\title{
Food as a Supplementary Cue Triggers Seasonal Changes in Aggression, but Not Reproduction, in Siberian Hamsters
}

\author{
Allison M. Bailey*, Nikki M. Rendon, Kyle J. O’Malley, and Gregory E. Demas \\ Department of Biology, Indiana University \\ 1001 E $3^{\text {rd }}$ St., Bloomington, IN, USA \\ *Corresponding author: allibail@indiana.edu \\ nrendon@indiana.edu \\ kylomall@indiana.edu \\ gdemas@indiana.edu
}

(C) 2016. This manuscript version is made available under the Elsevier user license http://www.elsevier.com/open-access/userlicense/1.0/ 


\section{Abstract}

Animals living in temperate regions prepare for harsh winter conditions by responding to environmental cues that signal resource availability (e.g., food, day length). Siberian hamsters (Phodopus sungorus) breed in long, summer-like days (LD, $>14 \mathrm{~h}$ light), i.e., photoperiods, and undergo robust gonadal regression and become more aggressive when exposed to short, winterlike photoperiods that signal impending limited resources $(\mathrm{SD},<10 \mathrm{~h}$ light). When hamsters are reared within an intermediate photoperiod (ID, 13.5h light), they are reproductively active, but undergo gonadal regression in response to mild food restriction (FR) over 6-12 weeks. We hypothesized that short-term (1-2 weeks) FR in an ID photoperiod would provide a signal of impending limited resources and initiate the seasonal increase in aggression typical of SD photoperiods, as well as alter reproductive behaviors in advance of gonadal regression. To test this, we housed male and female hamsters in LD or ID photoperiods, with ad libitum (AL) access to food or a $90 \%$-AL ration. We tested aggressive behavior after one week and reproductive behavior after two weeks, and subsequently monitored females for pregnancy and litter production. Both sexes displayed increased aggression in the ID-FR treatment. Untreated male intruders were less likely to ejaculate when paired with ID females during reproductive encounters. ID-FR males were undergoing gonadal regression after two weeks, but were more likely to have ejaculated. Female pregnancy and litter characteristics were unaltered by treatment: females were equally likely to achieve pregnancy and produce comparable litters across treatment groups. Collectively, we demonstrate that a signal of diminishing resources in an ID photoperiod is sufficient to trigger seasonal aggression, but that hamsters are reproductively resilient to inhibitory environmental cues in the short term. Broadly, our findings 
provide an important context for exploring seasonal changes in behavior and physiology from an ultimate perspective.

\section{Keywords}

Seasonality, gonadal regression, food restriction, phenology

\section{Introduction}

In order to maximize reproductive success, animals living in temperate climates undergo changes in behavior and physiology to coincide with extreme variations in resource availability. Many such species breed seasonally, restricting reproduction to times of year most favorable for survival of self and offspring (Bronson 1985; Bronson \& Heideman 1994; Goldman 2001). Rodents such as Siberian hamsters (Phodopus sungorus), in addition to adjusting breeding according to time of year, also display changes in aggressive behavior. Siberian hamsters are more aggressive in the non-breeding season, despite low circulating levels of gonadal steroids such as testosterone (Jasnow et al. 2000; Scotti et al. 2007), the primary physiological correlate of aggression in many species (reviewed in: Wingfield et al. 1987; Archer 2006; Soma et al. 2015).

Photoperiod is the primary environmental cue signaling time of year in Siberian hamsters and many other seasonally breeding animals. Photoperiod is encoded through a physiological signal of melatonin, which is secreted from the pineal gland during the night, in the absence of light (reviewed in: Lincoln et al. 2003). Greater durations of melatonin secretion in longer dark periods of winter (approximately 8-10 hours of light) trigger seasonal responses such as robust gonadal regression to curtail reproduction as well as increased aggressive behavior (Hoffmann 1982; Bartness et al. 1993; Jasnow et al. 2000; Goldman 2001; Scotti et al., 2007; Rendon et al. 
2015a). Hamsters detect the duration of melatonin release in the present as well as the change in the duration of release over time in order to predict future environmental conditions. Thus, shortening photoperiods trigger appropriate changes in reproductive physiology that take place over several weeks in order to prepare for harsh, resource-scarce conditions of winter. It is generally understood that photoperiod functions as a "noise-free" cue that reliably indicates the likelihood of future conditions, but it does not directly affect survival and reproductive success itself. Rather, seasonal species use photoperiod to predict the future status of factors such as precipitation, presence of conspecifics, and perhaps most critical, food availability (Baker 1938). Photoperiod can be conceptualized as an "initial predictive" cue, which is used to trigger broad physiological responses over time (gonadal regression), whereas the immediate status of factors such as food availability can act as "supplementary" cues to fine-tune behavior (Wingfield \& Farner 1980). Adequate food availability is essential to support energy-intensive reproductive processes, and it is reasonable to hypothesize that hamsters similarly increase aggression in response to shortening photoperiod in order to prepare to defend limited food resources, as food availability affects aggressive behavior in a variety of species (Lore et al. 1986; Desy et al. 1990; Maruyama et al. 2010; Foltz et al. 2015). This specific hypothesis, however, has not been investigated, nor have broader questions related to the relative timelines of behavioral and physiological seasonal shifts in response to changing combinations of initial predictive and supplementary cues.

Siberian hamsters housed in constant photoperiods of either greater than $14 \mathrm{~h}$ (summer) or less than $10 \mathrm{~h}$ (winter) of light per day in laboratory conditions show definitive physiological and behavioral states such that supplementary cues (e.g., food) do not trigger reproductive responses (Paul et al. 2009a; Paul et al. 2009b; Bailey et al. in review). In these conditions, 
although hamsters cannot track changes in photoperiod over time, photoperiod provides an accurate prediction of conditions in the near future. However, if hamsters develop within a constant, intermediate (13.5 h light) photoperiod occurring closer to the vernal and autumnal equinoxes in nature, photoperiod cannot act as an accurate predictor of future conditions without providing the additional information of change in duration of melatonin release. In these conditions, supplementary cues like food availability can trigger the initiation of gonadal regression (Paul et al. 2009a; Paul et al. 2009b; Bailey et al. in review). This laboratory paradigm can be used to address questions of how animals respond to dynamic changes in environmental conditions.

The goal of this study was to investigate how hamsters coordinate changes in behavior and physiology in response to fluctuating supplementary environmental cues. We compared changes in aggressive and reproductive behavior in response to short-term mild food restriction (FR) in male and female hamsters housed in either a long-day (LD), summer-like photoperiod or an intermediate (ID), fall-like photoperiod. We also investigated effects on reproductive success by monitoring females' pregnancies. We hypothesized that hamsters become more aggressive in winter-like photoperiods because short photoperiod is a signal that food will be limited in the future. We therefore predicted that only hamsters in the ID-FR treatment would show an increase in aggressive behavior. We further hypothesized that changes in reproductive behavior would occur in advance of gonadal regression, with the prediction that ID-FR hamsters would exhibit impaired reproductive behaviors; we predicted females to be especially responsive, as reproduction is primarily energy-limiting for females, and sex differences in reproductive responses to seasonal shifts have been shown in related species (Beery et al. 2007). Further, we predicted that the occurrence of pregnancy would be limited in ID-FR hamsters, and pregnancies 
that did occur would be characterized by smaller litter mass or fewer pups, through possible mechanisms of embryo resorption or infanticidal behavior.

\section{Materials and Methods}

\subsection{Animals and Housing}

Adult ( $>60$ days of age; specific ages ranged from 4 to 10 months) male and female Siberian hamsters were obtained from 31 litters produced by 10 breeding pairs in our long-day (LD) photoperiod (16:8 hour light:dark cycle, lights on at 0300 hours Eastern Standard Time, EST) breeding colony and from 23 litters produced by 14 breeding pairs in our intermediate-day (ID) photoperiod (13.5:10.5 hour light:dark cycle, lights on at 0130 hours Eastern Standard Time, EST) breeding colony at Indiana University. Animals were weaned at 18 days of age and subsequently housed either individually or with 1-4 same-sex littermates before entering the experiment in adulthood. Hamsters subject to experimental treatments (females: $n=60,30$ from each photoperiod; males: $n=60,30$ from each photoperiod) were individually housed in polypropylene cages $(27.5 \times 17.5 \times 13.0 \mathrm{~cm})$ with Sani-Chip ${ }^{\circledR}$ bedding material for one week

prior to the start of experimental treatments. These animals received ad-libitum access to food (Lab Diet 5001, PMI Nutrition) throughout development prior to the experiment and ad-libitum access to water at all times. Hamsters assigned as behavioral intruders (females: $n=25$ from LD photoperiod, $n=36$ from ID photoperiod; males: $n=35$ from LD photoperiod, $n=26$ from ID photoperiod) were housed in same-sex sibling pairs when possible, and in triplicate when needed, in polypropylene cages with the same dimensions and bedding material for one week prior to the first behavioral trial. Intruder hamsters received ad-libitum access to food and water at all times. Temperature and humidity were maintained at $20 \pm 2^{\circ} \mathrm{C}$ and $50 \pm 10 \%$, respectively. 
All animal procedures were performed in accordance with the National Institutes of Health Guide for the Care and Use of Laboratory Animals and were reviewed and approved by the Indiana University Bloomington Institutional Animal Care and Use Committee.

\subsection{Experimental Design}

Hamsters within each sex were randomized by breeding pair source and litter into 4 experimental groups, in a full factorial design by photoperiod (LD or ID) and food availability (ad-libitum, AL or food restriction, FR). All animals were weighed weekly. FR animals began receiving daily food rations after 1 week of individual housing and monitoring of baseline food intake, and continued receiving daily rations until time of euthanasia. AL animals' food intake was assessed weekly. After 1 week of FR, all animals participated in an aggressive behavior trial with a same-sex intruder. After 2 weeks of FR, all animals participated in reproductive behavior trials with an opposite-sex intruder nightly for up to 5 nights, to allow for females to display reproductive receptivity during their estrous cycle (see section 2.5). Male hamsters were euthanized for tissue collection immediately following a trial with a receptive female, or on the fifth night if the female did not display receptivity. Female intruder hamsters were vaginally lavaged to collect any sperm present in the vaginal canal immediately following the trial, and were subsequently euthanized. Female experimental hamsters were monitored for pregnancy following reproductive behavior trials for up to 20 days (gestation for first litters is 18 -19 days (Scribner \& Wynne-Edwards 1994)). Pregnant female hamsters that produced a litter were further monitored for infanticidal behavior for 5 days after birth, at which time the females and litters were euthanized. Non-pregnant females were euthanized for tissue collection after 
confirming the absence of a litter after 20 days. All females' uterine horns were stained to detect embryo implantation sites indicating pregnancy (see section 2.6).

\subsection{Food Restriction}

For 5 days immediately prior to the start of experimental treatments, ad libitum food intake was measured daily in each animal to assess individual levels of baseline intake. Each FR hamster was assigned a ration equal to $90 \%$ of their individual baseline intake, which was provided just prior to lights out each day (1500h EST for ID, 1900h EST for LD). FR continued throughout the monitoring of potentially pregnant females. To account for increased energetic needs during pregnancy and lactation, we consulted data from our laboratory indicating daily food intake of pregnant females throughout pregnancy and early lactation (French et al. 2016; Table 1). 


\begin{tabular}{|c|c|c|c|c|c|c|c|c|c|c|c|c|c|c|c|c|c|c|c|c|c|c|c|c|}
\hline Day of pregnancy/lactation & 1 & 2 & 3 & 4 & 5 & 6 & 7 & 8 & 9 & 10 & 11 & 12 & 13 & 14 & 15 & 16 & 17 & 18 & 19 & 20 & 21 & 22 & 23 & 24 \\
\hline Average AL intake & 2.8 & 2.8 & 2.4 & 2.9 & 3.1 & 3.4 & 3.2 & 3.6 & 3.8 & 4.2 & 4.6 & 4.4 & 5.0 & 4.8 & 4.7 & 4.8 & 5.0 & 6.0 & 6.8 & 7.8 & 8.4 & 8.2 & 11.3 & 9.3 \\
\hline $90 \%-A L$ ration & 2.5 & 2.5 & 2.2 & 2.6 & 2.8 & 3.0 & 2.9 & 3.2 & 3.4 & 3.8 & 4.1 & 4.0 & 4.5 & 4.3 & 4.2 & 4.3 & 4.5 & 5.4 & 6.1 & 7.1 & 7.6 & 7.4 & 10.2 & 8.4 \\
\hline
\end{tabular}

Table 1. Average ad libitum (AL) food intake calculated from 74 pregnant females in a previous experiment in our laboratory (French et al. 2016) and 90\% of each daily value, which was used to determine the daily ration for FR females during pregnancy monitoring in the present experiment. Pregnancy lasted from day 1 to day 18-19. Days 20-24 represent rations provided after litters were born, during lactation. 
Daily intake was averaged over 74 pregnant females, and $90 \%$ of each day's average (unless an individual's previously assigned FR ration was larger, in which case she received that ration to avoid extensive nutritional stress) was provided to each FR female, starting immediately following a receptive reproductive behavior trial, or following the fifth trial if the female was not receptive. FR continued throughout pregnancy and for 5 days after the birth of a litter. For all FR animals, upon providing daily rations, any remaining ration in the cage food hopper from the previous day was collected and weighed $( \pm 0.1 \mathrm{~g})$ to gain a more accurate approximation of actual intake, although it was not feasible to differentiate calorie consumption from hoarding or cheek pouch storage.

\subsection{Aggressive Behavior Trials}

Aggressive behavior was recorded and analyzed after 1 week of FR for same-sex social interactions according to previously outlined methods for males and females of this species, using a resident/intruder paradigm (Jasnow et al. 2000; Rendon et al. 2015a). Dyads were staged such that they were composed of a resident hamster (exposed to experimental treatments) and a same-sex intruder hamster. Residents were paired with intruders of approximately the same age, comparable mass $( \pm 5 \%)$, and from different parents. Photoperiod treatment was matched for hamster pairs (e.g., ID animal with ID animal) for the majority of the interactions; however, there was a small proportion of ID interactions (females: 10\%, males: 6.7\%) in which there was a mismatch.

Behavior trials were held in a dark procedure room under red light, within the first 2 hours of the dark period. Resident home cages were positioned on a laboratory bench in front of a video camera and flanked by two mirrors, to ensure visibility of all cage 
corners. The interaction commenced when the intruder was introduced into the home cage of the resident and animals were observed and recorded for 5 minutes, after which the intruder was removed and the interaction ended. Aggressive behaviors in the video files were then analyzed using ODLog ${ }^{\mathrm{TM}}$ (Macropod Software, Eden Prairie, MN) by an experienced observer blind to experimental treatment. In both sexes, we quantified attacks (latency to first attack, total number and duration) and chases (total number and duration) initiated by the resident hamster to assess aggression. A principal components analysis (PCA) was used on the aggression variables for males and females, and one component was extracted that explained $80.75 \%$ of the total variance in aggressive behavior (Supplementary Table 1).

\begin{tabular}{|c|c|}
\hline Aggression Variables & PC $_{\text {AGG }}$ \\
\hline Number of attacks & $\mathbf{0 . 9 8}$ \\
\hline Duration of attacks (s) & $\mathbf{0 . 9 5}$ \\
\hline Number of chases & $\mathbf{0 . 9 9}$ \\
\hline Duration of chases (s) & $\mathbf{0 . 9 2}$ \\
\hline Latency to first attack (s) & $\mathbf{- 0 . 6 0}$ \\
\hline Eigenvalue & 4.04 \\
\hline \% variance explained & 80.75 \\
\hline
\end{tabular}

Supplementary Table 1. Principal components loading and eigenvalues for male and female resident aggressive behaviors $\left(\mathrm{PC}_{\mathrm{AGG}}\right)$. Bold values indicate that all variables loaded strongly within the component $(-0.5$ or 0.5$)$.

All variables loaded strongly on the first component; therefore we used the composite aggression score $\left(\mathrm{PC}_{\mathrm{AGG}}\right)$ to examine the effects of photoperiod, food, and sex on overall aggression of resident hamsters. 


\subsection{Reproductive Behavior Trials}

Reproductive behavior trials took place after 2 weeks of FR, starting 30 minutes after lights out (1930h EST for LD, 1530h EST for ID). Each resident hamster was exposed to the same opposite-sex intruder nightly for up to 5 nights, to allow females to exhibit reproductive receptivity during their estrous cycles (it is not possible to predict female receptivity using vaginal epithelial cytology in this species (Erb et al. 1993; McMillan \& Wynne-Edwards 1999; Dodge et al. 2002)). Residents and intruders were paired as in the aggression trials, with comparable age and size, and from different parents. As in the aggression trials, most animals were matched by photoperiod treatment, but a small proportion (females: 6.7\%; males: 10\%) were mismatched.

Behavior trials were held in a dark procedure room under red light. Each night, each pair was placed inside a polypropylene cage $(27.5 \times 17.5 \times 13.0 \mathrm{~cm})$ with clean Sani-Chip ${ }^{\circledR}$ bedding and observed for up to 5 minutes. Cages were positioned on a laboratory bench in front of a video camera and flanked by two mirrors, to ensure visibility of all cage corners. If the male was able to mount the female successfully, or if the female showed obvious signs of reproductive receptivity (generally docile/compliant behavior, lordosis posture) during the 5 minute observation period, recording commenced immediately and continued for 30 minutes. If a female within a pair did not display signs of receptivity during the 5 minute observation period on any of the 5 nights, the pair was recorded for 5 minutes following the observation period on the fifth night. Reproductive behaviors in the video files were then analyzed using ODLog ${ }^{\mathrm{TM}}$ by an experienced observer blind to experimental treatment. 
Female reproductive behaviors for analysis included the occurrence of receptivity as well as number and duration of lordosis posture occurrences, and their male intruders were monitored for the occurrence of ejaculation. We also quantified the number of attacks females initiated toward their male intruders. A proportion (20\%) of the videos were monitored to determine if female scent marking behavior, in which the ventral gland is rubbed along the ground surface, should be quantified, but only 1 female performed 1 scent marking event, so further analysis was not conducted. Male reproductive behaviors for analysis included ejaculation (occurrence and latency), insemination (see section 2.7), and mounting (vaginally-directed mounts and mis-directed mounts). We also assessed the total duration of male ano-genital investigation of their female intruders, as well as number of scent markings.

\subsection{Pregnancy and Litter Monitoring}

Following reproductive behavior trials, females were retained in their assigned photoperiod and food treatment groups for at least 20 days to monitor for resulting pregnancies. FR food rations were provided as described above, AL intake was monitored weekly, and females were weighed weekly. Starting on day 15 after the receptive trial, each female was monitored every 24 hours for the presence of a litter. On the day of birth of each litter, pups were counted and weighed to assess litter mass at birth. Pups were counted each day for 5 days after birth to detect occurrences of infanticide. On day 5 , females were euthanized via a ketamine/xylaxine cocktail and pups were deeply anesthetized with isoflurane vapors and rapidly decapitated. Females that did not produce a litter were euthanized 20 days following the behavior trial. Reproductive 
tissues (ovaries, uterine horns, and perimetrial white adipose tissue (PWAT)) were collected from females at time of euthanasia to determine reproductive tissue mass. Uterine horns were stained to visualize embryo implantation sites by immersing them in a $10 \%$ ammonium sulfide solution for 10 minutes and rinsing with distilled water (Kopf et al. 1964; Roosa et al. 2015). Stained implantation sites appeared as dark spots along the uterine horns; uterine horns from non-pregnant females were devoid of spots (Supplementary Fig. 1).

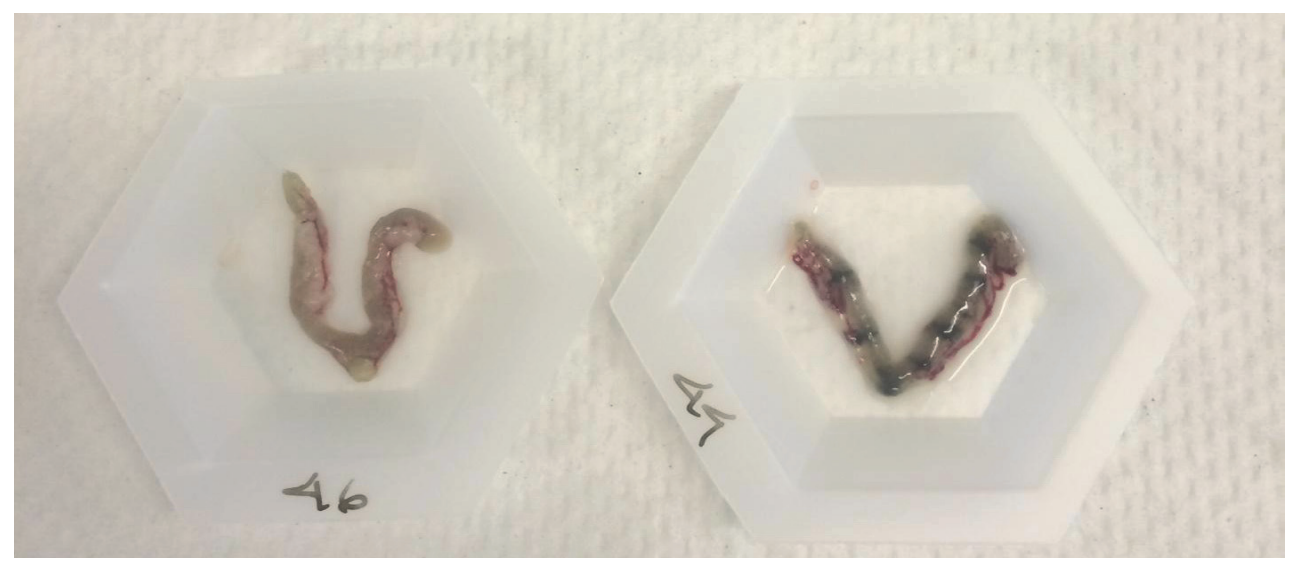

Supplementary Figure 1. Comparison of uterine horns post-ammonium sulfide staining in a non-pregnant female (left) and a pregnant female (right). The pregnant female depicted was determined to have 7 implantation sites ( 2 in the left horn, 5 in the right). 


\subsection{Male Insemination Assessment and Tissue Collection}

Immediately following the behavioral trial in which their female intruder was receptive, or on night 5 following recording, males were euthanized via a ketamine/xylaxine cocktail and tissues (testes, epididymal white adipose tissue (EWAT)) were collected to determine reproductive tissue mass. A sample of vaginal fluid was collected from each female intruder following the trial to determine whether the male had successfully inseminated her. $30 \mu \mathrm{L}$ of sterile $0.9 \%$ saline was lavaged through the vaginal canal 3-5 times, placed on a glass microscope slide, and allowed to dry. Once dry, samples were fixed in methanol and stained with a $10 \%$ Giemsa solution (SigmaAldrich ${ }^{\circledR}$ Procedure No. GS-10). Cells were visualized using a light microscope and the presence or absence of sperm was recorded.

\subsection{Statistical Analysis}

All statistical analyses were performed in JMP v. 11.0.0/12.0.1 (SAS Institute, Inc., Cary, NC) or SPSS v. 20.0/23.0.0 (IBM Corp., Armonk, NY), and attributed statistical significance at $p<0.05$. Sample sizes totaled 15 hamsters per sex per photoperiod/food treatment combination, except for week 2 measurements in ID-FR females; two hamsters died spontaneously, unrelated to experimental procedures, between week 1 and 2. Data distributions were checked for homogeneity of variance and for normality of model residuals; those distributions with unequal variances or producing non-normal residuals were transformed to best meet these assumptions for parametric tests. Food intake data were square root-transformed, except for male baseline food 
intake, which could not meet parametric assumptions, and was analyzed using a nonparametric Wilcoxon rank-sum test. Attacks (number and duration), number of chases, and latency to attack (aggression trial) were square root-transformed, and duration of chases was log-transformed. Male mounting and ano-genital investigation behavior data were square root-transformed, and male scent marking behavior data and female attack (reproduction trial) data were log-transformed. Ovary and PWAT mass, and the number of pups lost to infanticide within litters were log-transformed. EWAT mass could not be transformed to meet parametric assumptions, and was analyzed using the Wilcoxon test. Two females were excluded from food intake analysis: one ID-AL female had abnormally low baseline food intake, but exhibited normal AL intake during the experiment. Another ID-AL female showed exceedingly high weekly intake, so was determined to be hoarding and not appropriate to include in intake analysis. Three females were excluded from uterine horn mass analysis: one (ID-AL) was weighed on day 1 after birth (entire litter was consumed within the first day) and was thus extremely large, one (LD-AL) was mistakenly discarded following ammonium sulfide staining, and the third (LD-FR) was abnormally large. This latter female was similarly excluded from ovary mass analysis because of a growth on one ovary that resulted in an abnormally large ovary mass, which may also have contributed to her abnormal uterine horn mass. For measurements of body mass and food intake over time, a repeated measures analysis of variance (ANOVA) was used to detect effects of photoperiod and food treatments within and between subjects of each sex, with time as a within-subjects variable. Withinsubjects comparisons for female and male body mass as well as pregnant female and male food intake violated assumptions of sphericity and were Greenhouse-Geiser (G-G) 
corrected. Within time points of data collected over time (individual weeks for body mass/food intake), we performed two-way ANOVAs to detect effects of photoperiod, food, and the interaction of photoperiod $\mathrm{x}$ food, followed by Tukey's HSD post-hoc comparisons if there were statistically significant effects. For aggressive behaviors, we tested the effects of photoperiod and food, and also the effects of photoperiod and sex on resident hamsters. We used a two-way analysis of variance (ANOVA) to examine all aggression variables (i.e. $\mathrm{PC}_{\mathrm{AGG}}$, number and duration of attacks and chases, and latency to first attack), followed by Tukey's HSD post-hoc comparisons if there were statistically significant effects. Reproductive measurements expressed as proportions (female receptivity, ejaculation/insemination, pregnancy, and litter production) were assessed using a logistic regression including photoperiod and food in a full factorial design, as well as $\chi^{2}$ contingency table analysis to detect differences among groups. All other measurements (\% change in body mass; reproductive mass; reproductive behaviors (in males: number and duration of vaginally-directed/mis-directed/total mounts, latency to ejaculate, duration of ano-genital investigation, number of scent markings; in females: duration of lordosis display and number of attacks); number of implantation sites; number of pups on day of birth, on day 5, and lost to infanticide; and litter mass) were assessed through two-way ANOVAs including photoperiod and food in a full factorial design, followed by Tukey's HSD post-hoc comparisons if there were statistically significant effects. Final body mass was included as a significant covariate in the model for paired testes mass, paired ovaries mass, PWAT mass, and litter mass on day of birth. Pregnancy status was included as a significant covariate in the model for PWAT mass; PWAT mass in pregnant/non-pregnant females was then assessed using Student's $t$-test. 


\section{Results}

\subsection{Body Mass, Food Intake, and Reproductive Mass}

Female baseline body mass differed according to photoperiod, with ID females having lower body mass than LD females initially $\left(F_{3,56}=9.65, p<0.001\right)$. Over the two weeks of experimental treatments, ID-AL females gained body mass and LD-FR females lost body mass (between subjects photoperiod effect: $F_{1,56}=21.31, p<0.001$; within subjects time x photoperiod effect, G-G corrected: $F_{1,76}=5.87, p=0.011$; within subjects time x food effect, G-G corrected: $F_{1,76}=6.87, p=0.0055$, G-G corrected) (Fig. 1a). These changes in mass resulted in statistically significant differences between ID-AL and LD-FR female groups in total percent change in body mass $\left(F_{3,56}=4.40, p=0.0076\right)$ (Fig. 1b). In contrast, male baseline body mass was similar across groups $\left(F_{3,56}=0.45, p\right.$ $=0.72$ ), there were no effects of treatment between individuals over time, and only time $\mathrm{x}$ food contributed to changes in mass within individuals $\left(F_{1,80}=14.30, p<0.001\right.$, G-G corrected) (Fig. 1a). Food treatment affected total percent change in mass in males $\left(F_{1,56}\right.$ $=16.05, p<0.001)$, but FR males lost a significant percentage of their baseline body mass compared to their AL counterparts only in the LD photoperiod, not in the ID photoperiod (Fig. 1b).

In females and males, baseline food intake differed across photoperiods, with ID hamsters consuming more than LD hamsters (females: $F_{1,54}=11.17, p=0.0015$; males: $Z$ $=2.10, p=0.036$ ). In both sexes, hamsters assigned to FR groups differed in baseline intake between photoperiods, with ID-FR animals consuming more than LD-FR animals (females: $F_{4,54}=3.71, p=0.0097$; males: $Z=2.28, p=0.023$ ) (Fig. 1c). Over time, there 
were no treatment effects between individuals in females, but food intake over time within individuals varied according to food treatment, with FR animals consuming less food, as expected (within subjects time $\mathrm{x}$ food effect: $F_{2,53}=21.79, p<0.001$ ). Food treatment similarly affected changes in intake within male individuals (within subjects time $\mathrm{x}$ food effect, G-G corrected: $\left.F_{1,79}=15.88, p<0.001\right)$. Change in intake over time also varied according to photoperiod $\left(F_{1,56}=5.75, p=0.020\right)$ and food $\left(F_{1,56}=4.04, p=\right.$ 0.049) between individuals in males (Fig. 1c). In both sexes, AL intake in both photoperiods declined after week 1 such that there were no differences in intake among groups in week 2 (females: $F_{3,55}=1.24, p=0.30$; males: $F_{3,56}=2.48, p=0.071$ ) (Fig. 1c).

At the end of week 2, gonadal regression in ID-FR males was underway, with paired testes mass measuring significantly lower in this group compared to LD groups $\left(F_{4,55}=5.34, p=0.0011\right)($ Fig. $1 \mathrm{~d})$. EWAT mass did not differ among groups $\left(\chi^{2}(3)=\right.$ 2.70, $p=0.44)$ (Supplementary Table 2). In females, a significant treatment effect of photoperiod led to ID females having smaller uterine horns than LD females $\left(F_{1,5 l}=4.82\right.$, $p=0.033$ ) (Supplementary Table 2). Paired ovary mass and PWAT mass were unaffected by treatment (ovaries: $F_{4,52}=1.99, p=0.11$; PWAT: $F_{5,52}=1.87, p=0.15$ ) (Supplementary Table 2). Pregnancy significantly affected PWAT mass, with pregnant females having smaller PWAT mass than non-pregnant females $(t(45)=-6.53, p<0.001)$ (Supplementary Table 2). Paired ovary mass and uterine horn mass were unaffected by pregnancy (ovaries: $t(37)=-1.34, p=0.09$; uterine horns: $t(47)=1.10, p=0.14$ ) (Supplementary Table 2). 


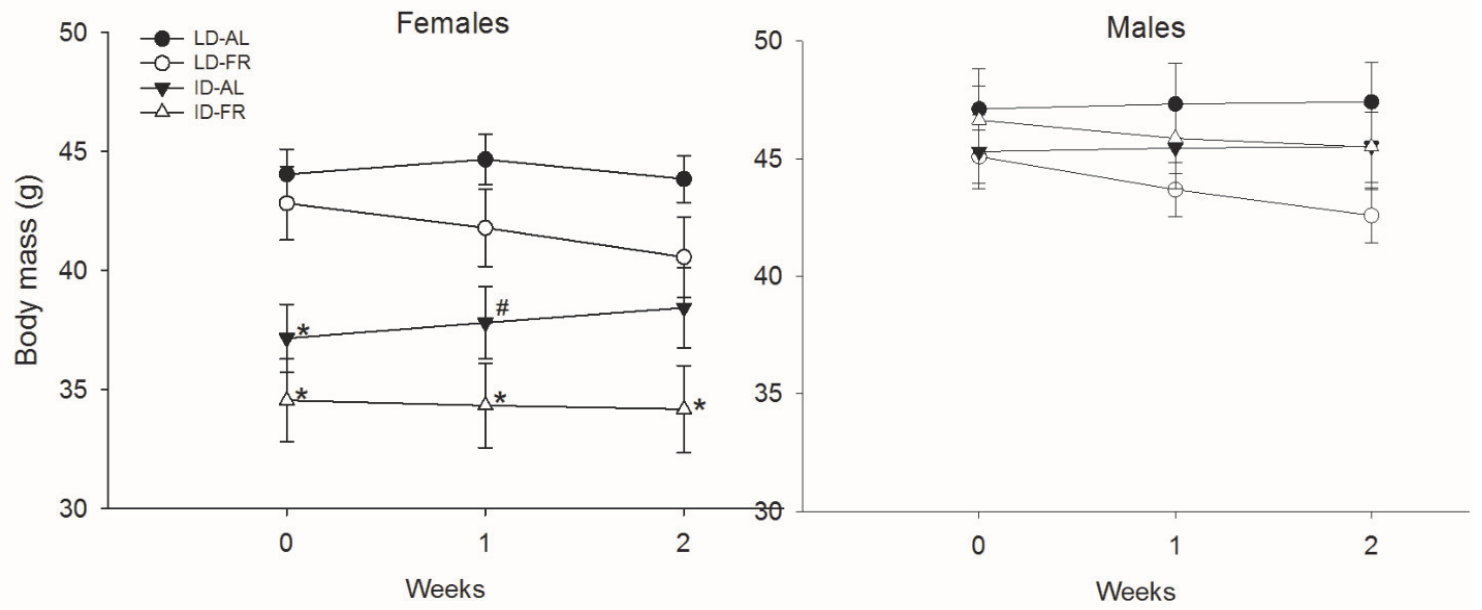

b 


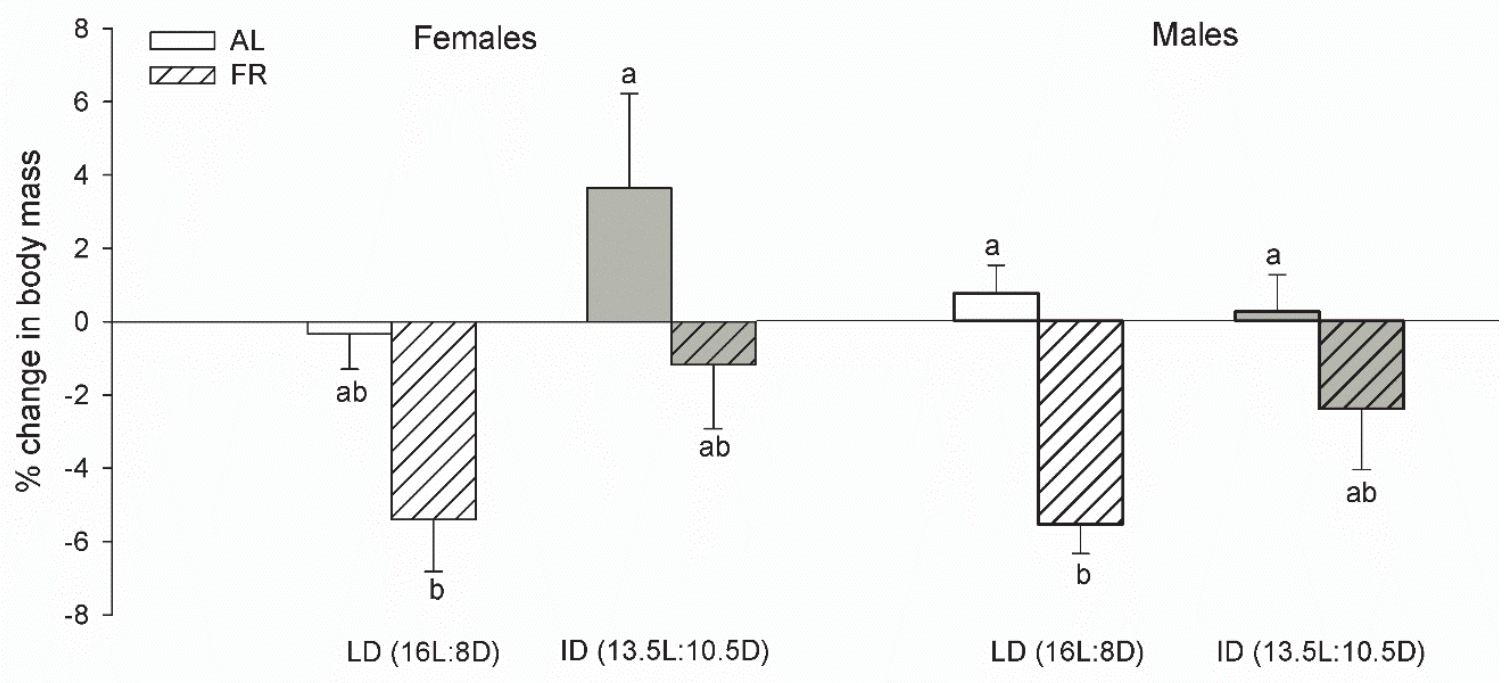

c

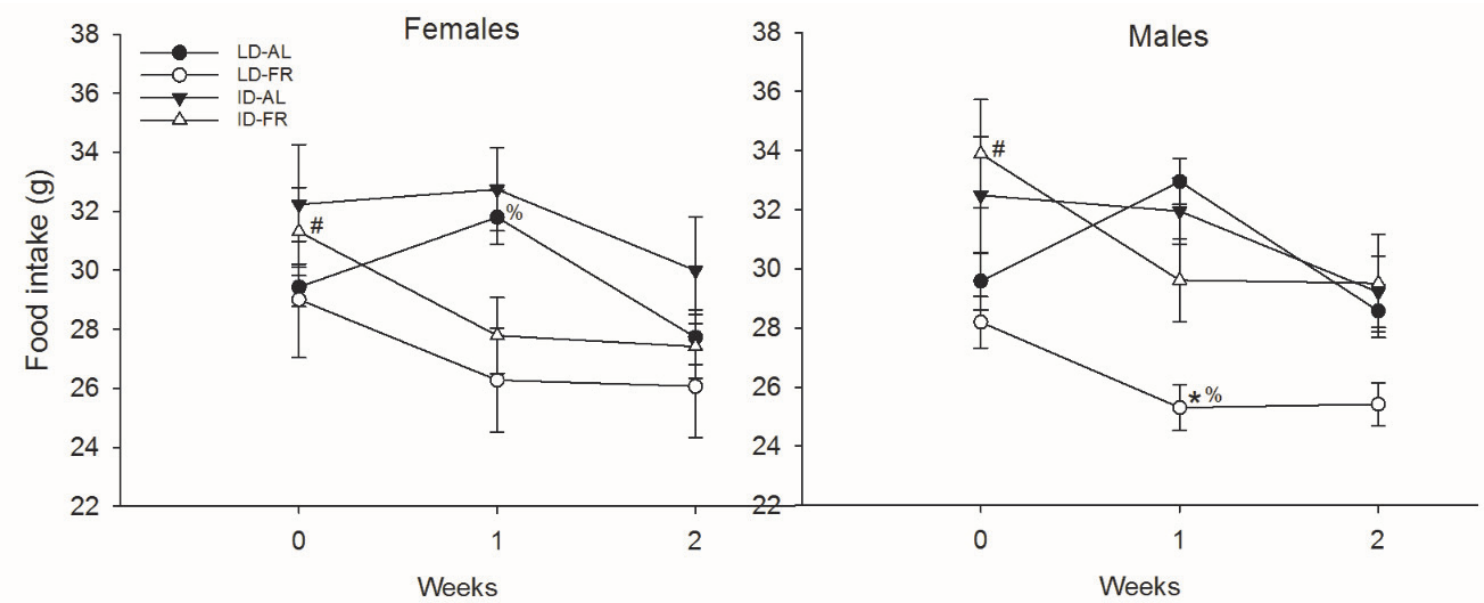

d 


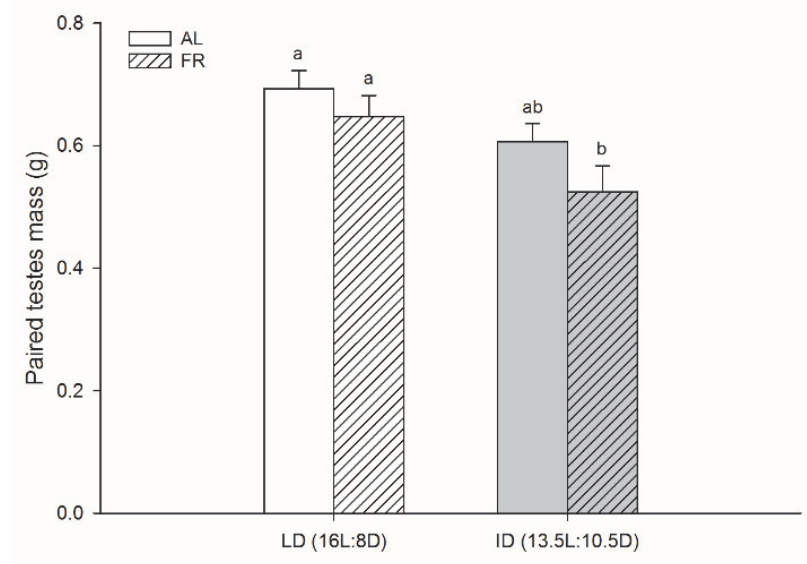

Figure 1. Mean ( \pm SEM) (a) body mass over time, (b) total percent change in body mass, and (c) food intake over time in male and female Siberian hamsters, and (d) paired testes mass of male hamsters after 2 weeks of photoperiod and food treatments. (a) (c) ${ }^{*} p<0.05$ versus both groups in opposite photoperiod, ${ }^{\#} p<0.05$ versus opposite photoperiod, same food treatment group, ${ }^{\%} p<0.05$ versus opposite food, same photoperiod treatment group. (b) (d) groups with different letters indicate statistically significant differences between group means $(p<0.05)$; groups sharing the same letter are statistically equivalent.

\begin{tabular}{|c|c|c|c|c||c|c|}
\hline Female tissues & LD-AL & LD-FR & ID-AL & ID-FR & Non-pregnant & Pregnant \\
\hline Ovaries (mg) & $8.7 \pm 0.7$ & $8.6 \pm 0.8$ & $9.3 \pm 0.8$ & $7.5 \pm 0.5$ & $8.9 \pm 0.4$ & $8.0 \pm 0.7$ \\
\hline Uterine horns (g) & $0.16 \pm 0.01$ & $0.14 \pm 0.01$ & $\mathbf{0 . 1 2} \pm \mathbf{0 . 0 1}^{\mathbf{*}}$ & $\mathbf{0 . 1 2} \pm \mathbf{0 . 0 2}$ & $0.13 \pm 0.01$ & $0.15 \pm 0.01$ \\
\hline PWAT (g) & $0.15 \pm 0.03$ & $0.13 \pm 0.03$ & $0.09 \pm 0.02$ & $0.15 \pm 0.04$ & $0.17 \pm 0.02$ & $\mathbf{0 . 0 5}^{\boldsymbol{0}} \pm \mathbf{0 . 0 1}^{\#}$ \\
\hline Male tissues & LD-AL & LD-FR & ID-AL & ID-FR & & \\
\hline EWAT (g) & $1.07 \pm 0.06$ & $0.90 \pm 0.04$ & $1.02 \pm 0.11$ & $1.03 \pm 0.09$ & & \\
& & & & &
\end{tabular}


${ }^{*}$ Uterine horns significantly smaller in ID females than in LD females $(p<0.05)$

${ }^{*}$ PWAT in pregnant females significantly smaller than in non-pregnant females $(p<0.05)$

Supplementary Table 2. Mean $( \pm$ SEM) reproductive tissue mass from female (ovaries, uterine horns, and perimetrial white adipose tissue (PWAT)) and male (epididymal white adipose tissue (EWAT)) Siberian hamsters post-necropsy. Female tissues are shown organized by experimental treatment group (pregnancy status combined) as well as by pregnancy status (all 4 groups combined). Tissues from non-pregnant females were collected after confirming the absence of a litter (20 days post-reproductive behavior encounter) and tissues from pregnant females were collected 5 days post-birth. All FR females received adjusted (increased) FR rations between the reproductive behavior encounter and necropsy to account for possible pregnancy. EWAT and testes (Fig. 1) were collected from males immediately following the reproductive behavior encounter.

\subsection{Aggressive Behavior}

Female and male hamsters in the ID-FR treatment displayed increased overall aggression when compared to ID-AL animals well as animals in both $\mathrm{LD}$ groups $\left(\mathrm{PC}_{\mathrm{AGG}}\right.$, females: $F_{3,56}=4.52, p=0.007$; males: $F_{3,56}=4.98, p=0.003$ ) (Fig. 2a). Specifically, ID-FR females displayed more $\left(F_{3,56}=6.10, p=0.001\right)$ and longer $\left(F_{3,56}=3.93, p=\right.$ $0.01)$ attacks and more $\left(F_{3,56}=4.47, p=0.005\right)$ and longer $\left(F_{3,56}=3.20, p=0.03\right)$ chases. ID-FR males displayed more attacks $\left(F_{3,56}=5.49, p=0.002\right)$ and longer chases $\left(F_{3,56}=7.53, p=0.01\right)$. ID-FR males, unlike ID-FR females, did not display more chases $\left(F_{3,56}=1.10, p=0.36\right)$ or longer attacks $\left(F_{3,56}=1.08, p=0.37\right)$. Latency to first attack did not differ between groups (females: $F_{3,56}=6.69, p=0.23$; males: $F_{3,56}=2.34, p=$ $0.15)$. 


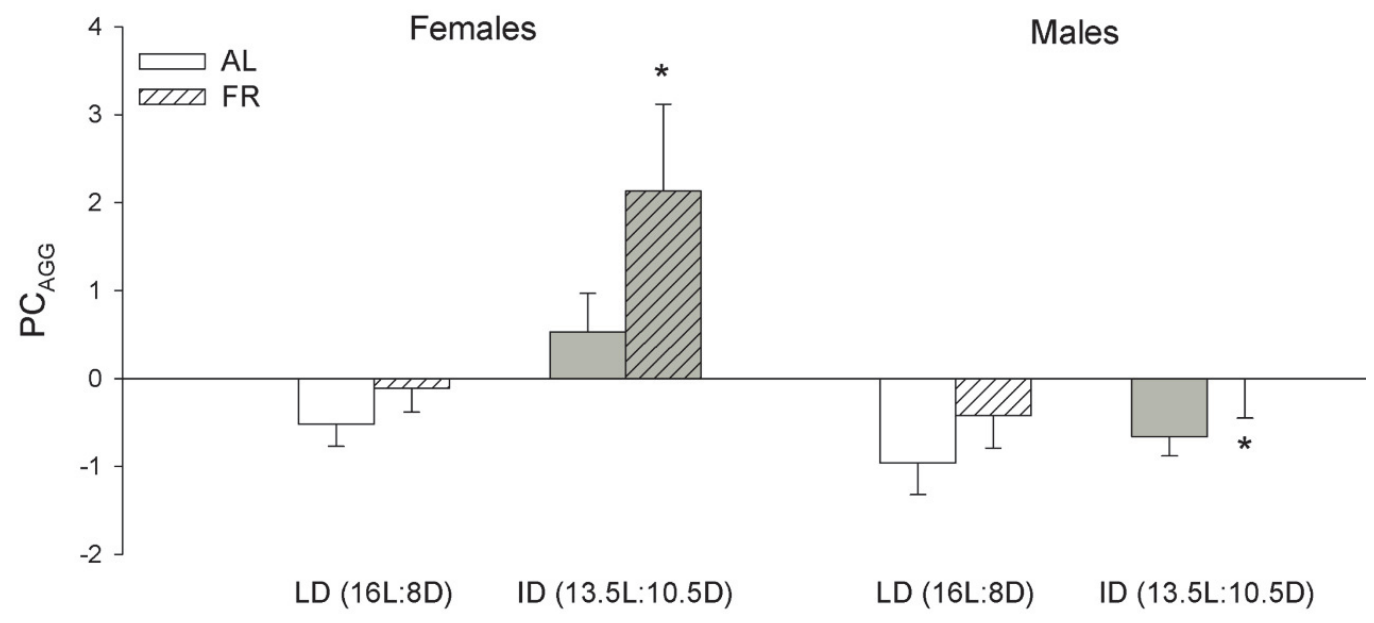

Figure 2. Mean ( $\pm \mathrm{SEM}$ ) overall aggression score $\left(\mathrm{PC}_{\mathrm{AGG}}\right)$ observed in male and female Siberian hamsters during the aggressive behavior encounter at the end of 1 week of photoperiod and food treatments. $p<0.05$ compared to other groups within sex.

\subsection{Reproductive Behavior}

Females were equally likely to display reproductive receptivity across treatment groups $\left(\chi^{2}(3)=4.35, p=0.23\right.$ ) (Fig. 3a). There were also no treatment effects or differences between groups for lordosis behavior (duration: $F_{3,35}=0.50, p=0.69$ (Fig. 3b); number: $F_{3,35}=1.08, p=0.37$ ). Male intruders, however, were less likely to ejaculate in the ID photoperiod $\left(\chi^{2}(1)=4.65, p=0.031\right)$ (Fig. $\left.3 \mathrm{c}\right)$. 
Males were equally likely to be paired with a receptive female intruder across treatment groups $\left(\chi^{2}(3)=5.15, p=0.16\right)$. A significant photoperiod $\mathrm{x}$ food interaction effect suggested an increased proportion of males achieving ejaculation in the ID-FR group $\left(\chi^{2}(1)=4.91, p=0.027\right)$, but a comparison across groups revealed no significant differences $\left(\chi^{2}(3)=6.56, p=0.087\right)$ (Fig. 3d). Although the ID-FR group had the highest proportion of males ejaculating, there were equal proportions of ejaculating males that produced sperm recovered from their female intruder across all groups $\left(\chi^{2}(3)=1.60, p=\right.$ 0.66 ) (Fig. 3e). Latency to ejaculate was also equivalent across groups $\left(F_{3,18}=0.52, p=\right.$ 0.67) (data not shown). Duration and number of mounting behaviors was robust to experimental treatments, with no treatment effects or differences among groups observed (duration of vaginally-directed mounts: $F_{3,38}=0.48, p=0.70$; number of vaginallydirected mounts: $F_{3,38}=0.17, p=0.92$; duration of mis-directed mounts: $F_{3,38}=0.48, p=$ 0.70 , number of mis-directed mounts: $F_{3,38}=0.24, p=0.87$; duration of total mounts: $F_{3,38}=0.27, p=0.85$; number of total mounts: $F_{3,38}=0.29, p=0.83$ ) (Fig. 3f). 

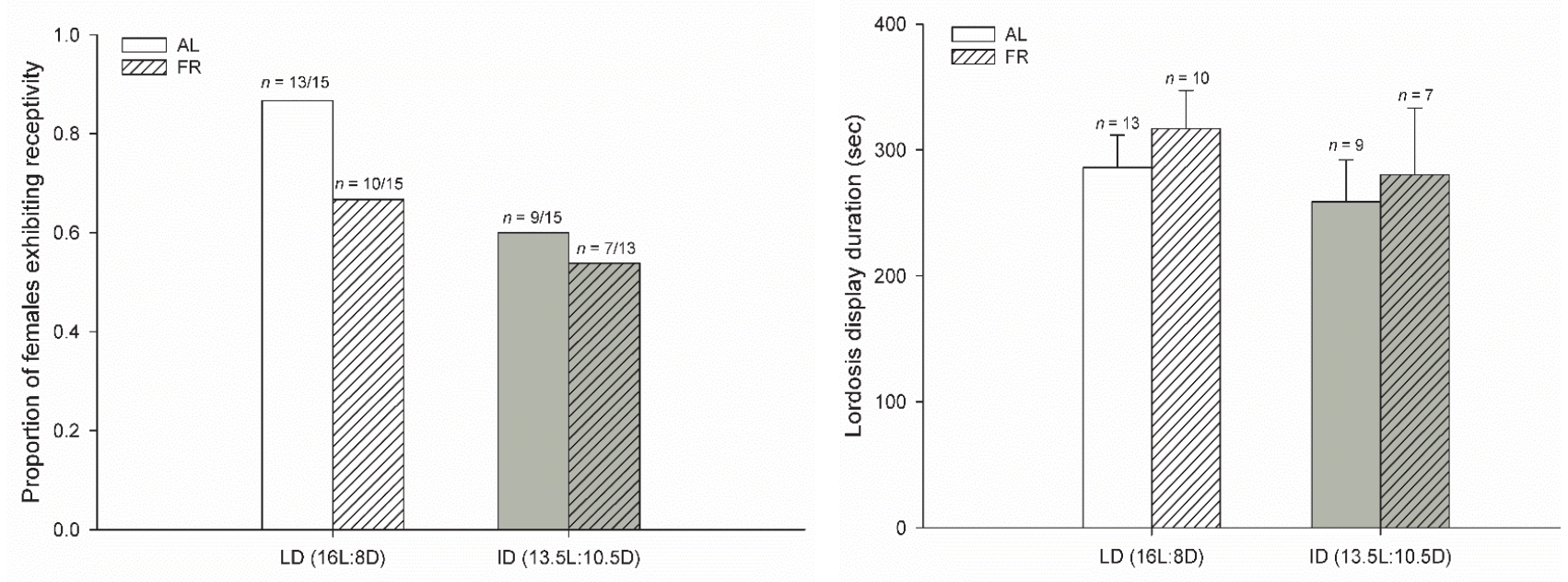

c

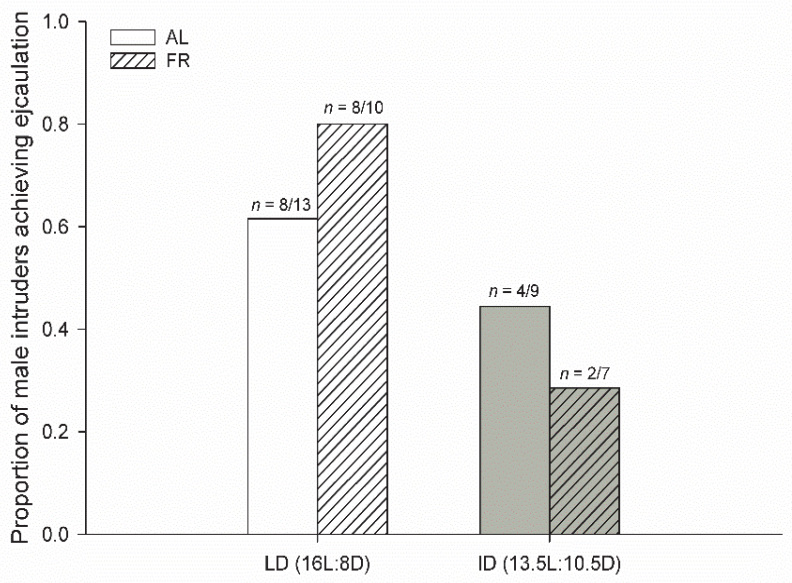

e d

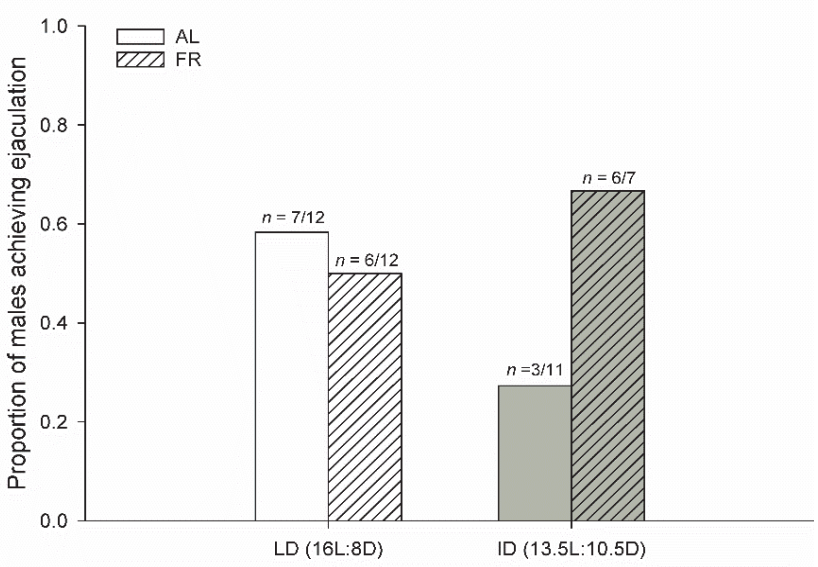



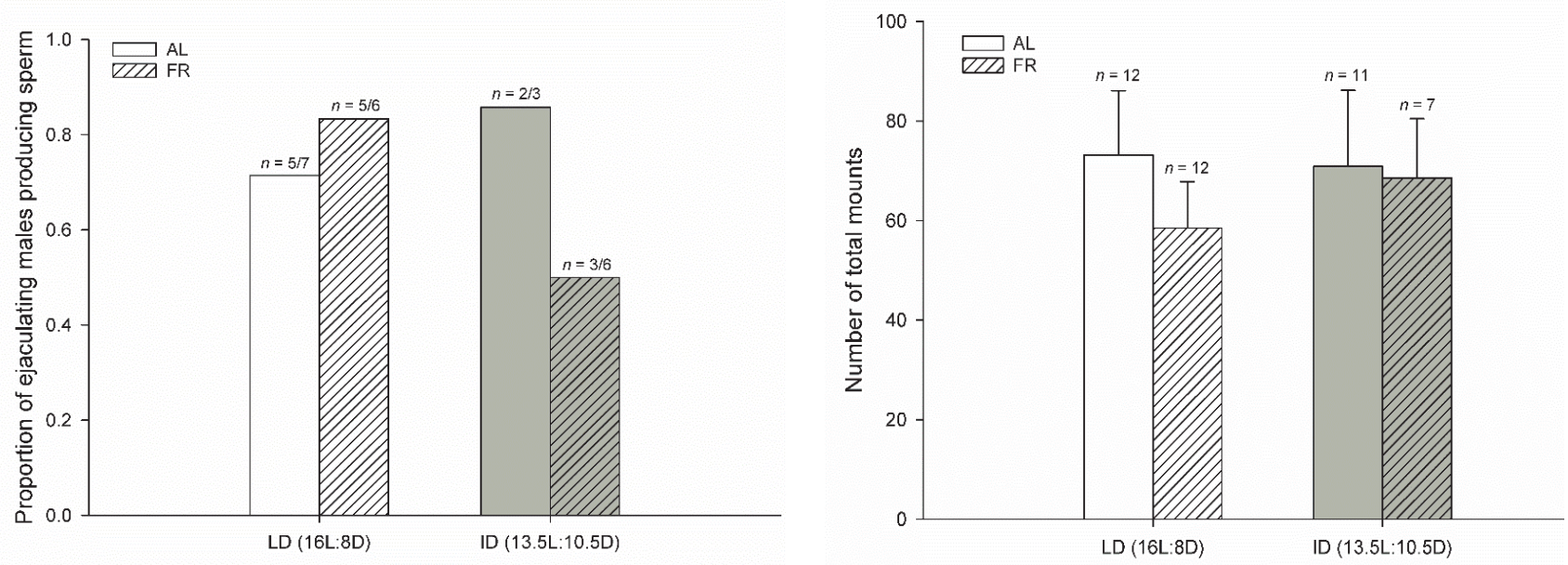

Figure 3. (a) Proportion of females exhibiting reproductive receptivity, (b) mean \pm SEM total duration of lordosis posture performed by receptive females, (c) proportion of male intruders achieving ejaculation when paired with experimental females; proportion of males (d) ejaculating and (e) producing sperm, and (f) mean \pm SEM total number of mounts (both vaginally-directed and mis-directed) performed by males, all observed during the reproductive behavior encounter after 2 weeks of photoperiod and food treatments. (c) Photoperiod significantly affected the proportion of male intruders paired with experimental females achieving ejaculation $(p<0.05)$. (d) A photoperiod $\mathrm{x}$ food interaction significantly affected proportion of males ejaculating $(p<0.05)$. There were no other treatment effects or post-hoc differences observed between groups for any other behaviors. Sample sizes are dependent on female receptivity rates.

FR females tended to attack their male intruder more often than AL females, but this effect fell short of significance $\left(F_{3,35}=3.79, p=0.060\right)$ (Fig. 4a). Food treatment had a significant effect on male ano-genital investigation of female intruders, with FR males performing less investigation $\left(F_{1,38}=4.82, p=0.034\right)$, but this effect failed to produce differences between treatment groups $\left(F_{3,38}=2.58, p=0.068\right)$ (Fig. 4b). Males also displayed scent marking behavior, but this behavior was unaffected by treatment $\left(F_{3,38}=\right.$ $1.28, p=0.30)($ Fig. 4c). 


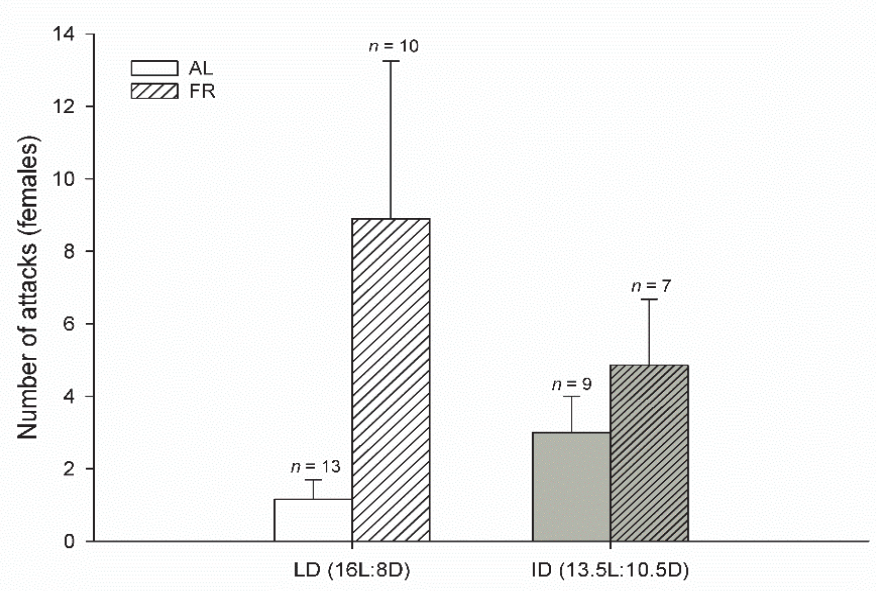

c

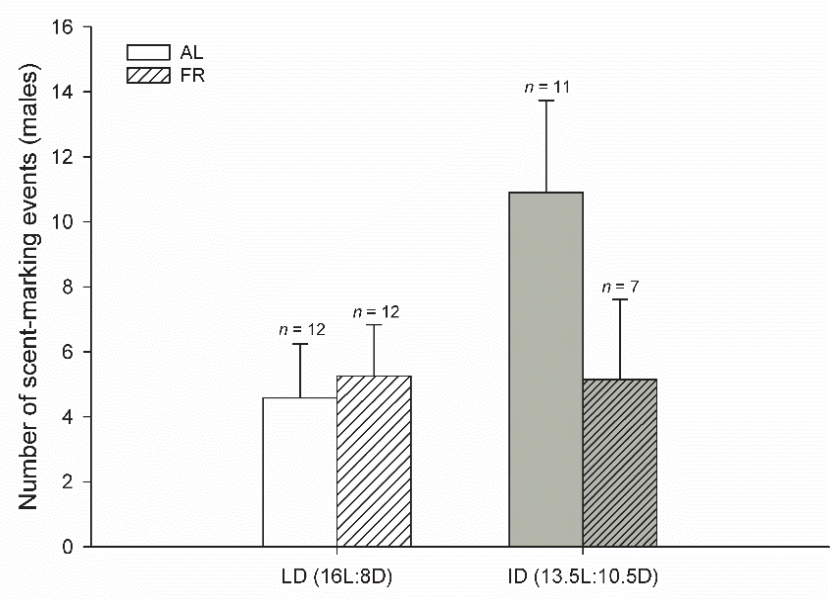

b

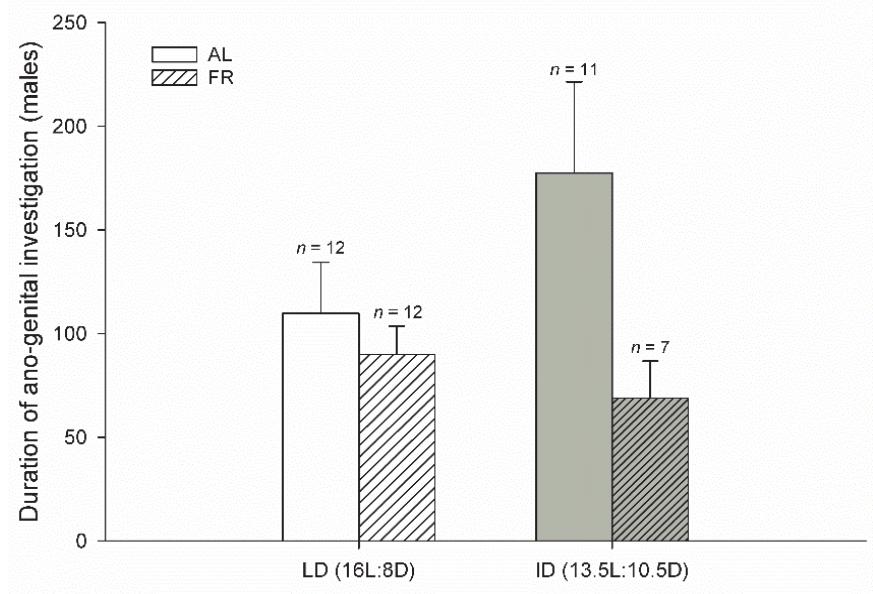

Figure 4. Mean ( \pm SEM) (a) number of attacks performed by females, (b) duration of ano-genital investigation performed by males, and (c) number of scent-marking events performed by males during the reproductive behavior encounter after 2 weeks of photoperiod and food treatments. (b) Food treatment significantly affected the duration of male ano-genital investigation of the female intruder $(p<0.05)$. There were no other treatment effects or post-hoc differences between groups observed for any other behaviors, other than a trend toward food affecting the number of attacks performed by females toward male intruders $(\mathrm{a}, p=0.06)$. Sample sizes are dependent on female receptivity rates.

\subsection{Pregnancy Outcomes}


The proportion of females (paired with a male that ejaculated) achieving pregnancy was equivalent across groups $\left(\chi^{2}(3)=1.35, p=0.72\right)$ (Fig. 5a). These pregnant females had equivalent numbers of implantation sites discovered in their uterine horns $\left(F_{3,18}=0.19, p=0.90\right)$ (Fig. $\left.5 \mathrm{~b}\right)$ and an equivalent proportion of pregnant females produced litters successfully across groups $\left(\chi^{2}(3)=2.79, p=0.43\right)$. Litter characteristics were also robust to treatments: there were no treatment effects or differences between groups for number of pups on day of birth $\left(F_{3,13}=1.63, p=0.23\right)$ (Fig. $\left.5 \mathrm{c}\right)$, number of pups lost to infanticide $\left(F_{3,13}=0.31, p=0.82\right)$ (Fig. 5 d), number of pups on day $5\left(F_{3,13}=\right.$ $0.44, p=0.73)$, or litter mass on day of birth $\left(F_{4,12}=0.58, p=0.64\right)$.
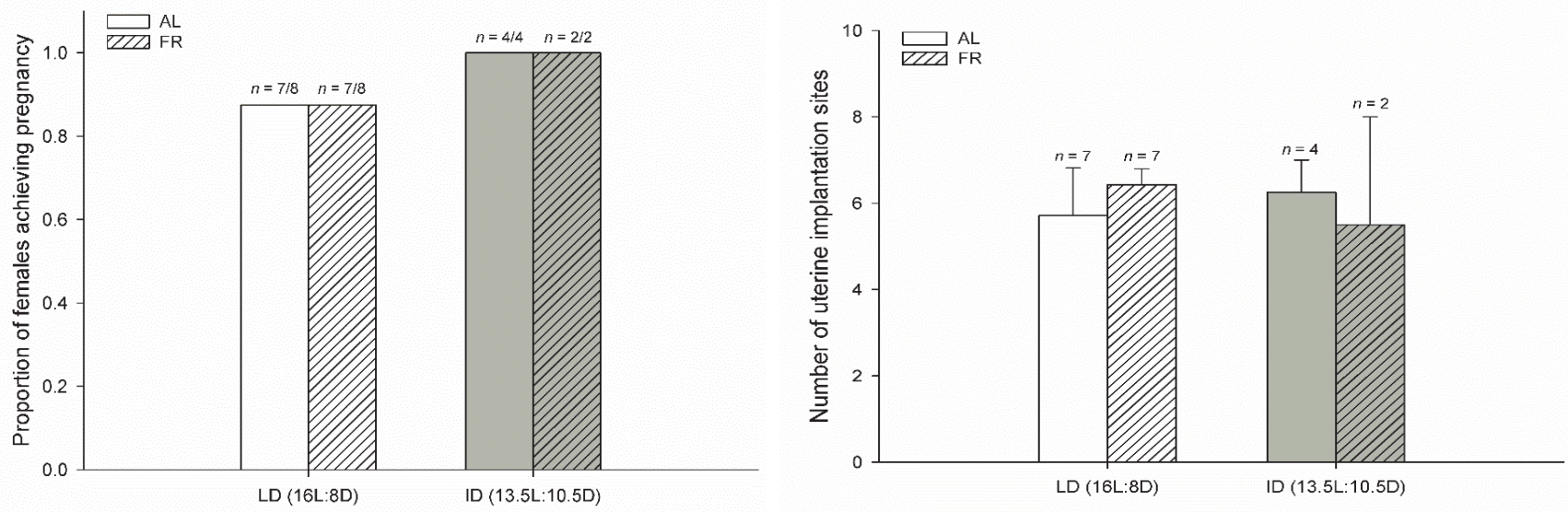

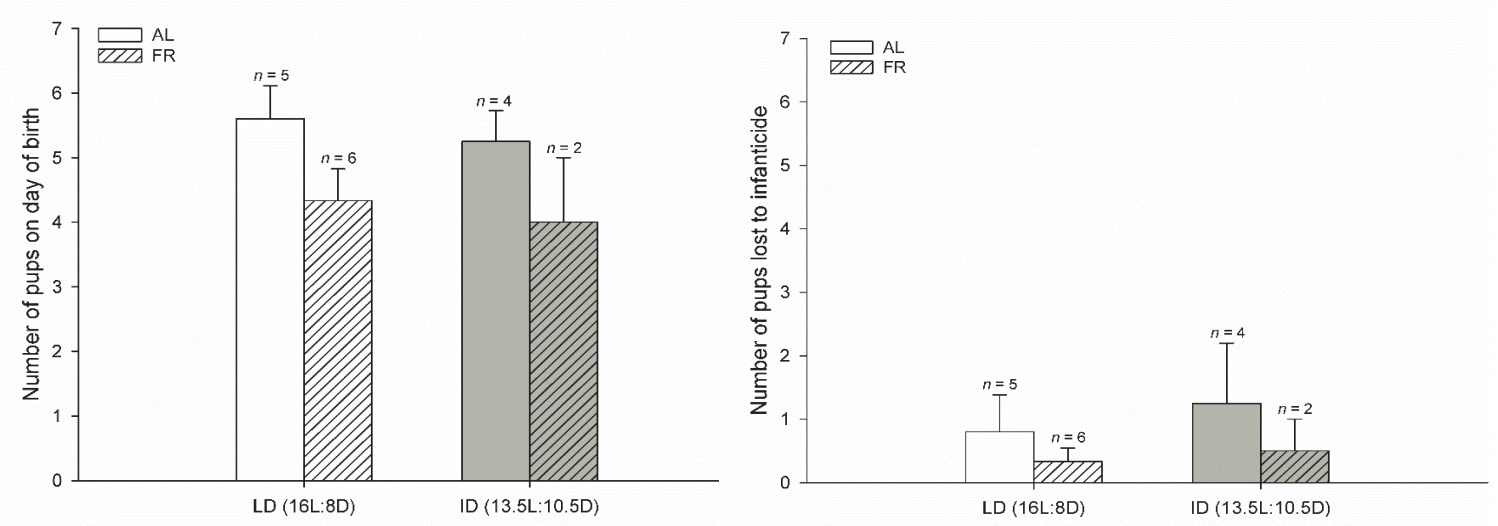

Figure 5. (a) Proportion of females achieving pregnancy after the reproductive behavior encounter and mean \pm SEM (b) number of implantation sites observed in uterine horns, (c) number of pups on day of birth, and (d) number of pups lost due to infanticide in the first 5 days after birth. No treatment effects or post-hoc differences between groups were observed. Sample sizes are dependent on male intruder ejaculation, pregnancy, and littering rates.

Body mass and food intake in pregnant vs. non-pregnant females were measured weekly during the experiment, rather than according to the day each female exhibited receptivity, and thus did not coincide with stage of pregnancy across individuals. Body mass changed between individuals according to photoperiod treatment $\left(F_{1,53}=13.37, p<\right.$ $0.001)$ and whether a litter was produced $\left(F_{1,53}=4.97, p=0.030\right)$, with litter-producing females tending to decline in body mass over time; however, these effects did not produce any post-hoc differences between experimental groups according to litter production at specific time points (week $4: F_{7,50}=1.86, p=0.096$; week $5: F_{7,50}=1.30, p$ $=0.27$; week 6: $F_{7,50}=1.83, p=0.10$ ) (Supplementary Fig. 2a). Within individuals, only photoperiod treatment affected changes in body mass, such that LD females appeared to 
lose body mass over time while ID females maintained constant mass (time $\mathrm{x}$ photoperiod effect, G-G corrected: $F_{2,119}=8.17, p<0.001$ ) (Supplementary Fig. 2a). Food intake changed over time within individuals according to food treatment and litter production (time $\mathrm{x}$ food effect, G-G corrected: $F_{3,195}=6.16, p<0.001$; time $\mathrm{x}$ litter effect, G-G corrected: $F_{3,195}=23.30, p<0.001$ ) (Supplementary Fig. $2 b$ ). There were no effects of treatment on food intake over time between individuals $\left(F_{4,50}=1.07, p=0.38\right)$. In the final week of the experiment, AL-treated females with litters consumed significantly more food than all other groups $\left(F_{7.47}=7.84, p<0.001\right)$ (Supplementary Fig. 2b).

a

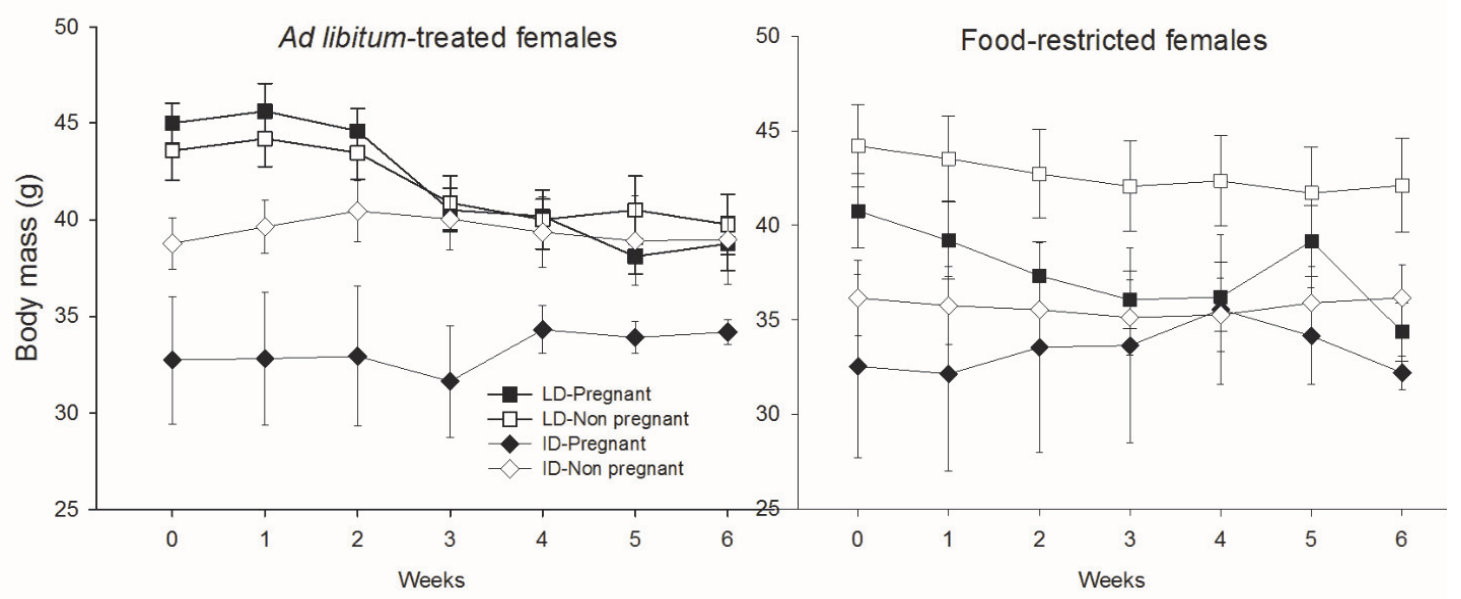




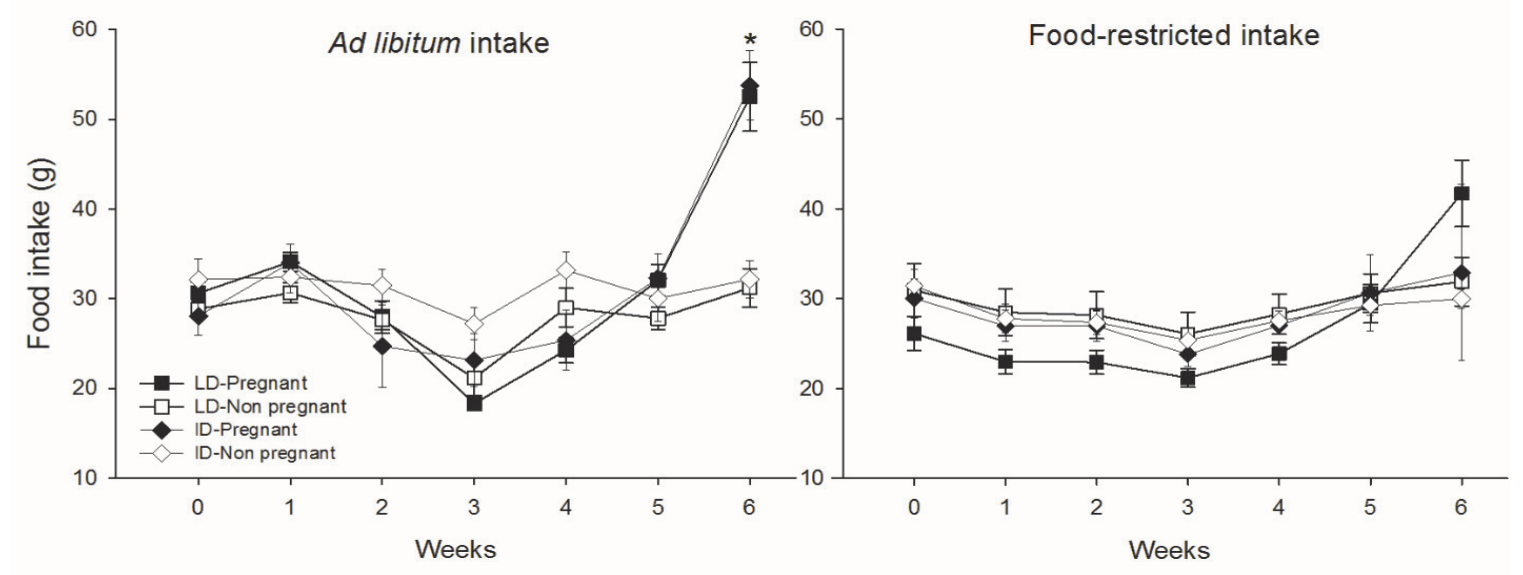

Supplementary Figure 2. Mean ( \pm SEM) (a) body mass over the entire experiment in females that were determined to have either produced a litter or did not produce a litter and (b) food intake over the entire experiment in litter-producing or non-litter-producing females of ad libitum (AL) and restricted (FR) food treatment groups. Data were collected according to the weekly experimental schedule rather than uniform times during pregnancy across females, resulting in sampling of pregnant females at various time points during pregnancy. For this reason, patterns in body mass between pregnant and non-pregnant females are difficult to discern, with no differences between groups in the weeks following the reproductive behavior trial (a). AL litter-producing females consumed significantly more food in the final week of the experiment compared to AL females that did not produce litters in both photoperiods $\left({ }^{*} p<0.05\right)$, but this difference did not exist significantly in FR females (b).

\section{Discussion}

The goal of the present study was to examine whether short-term, mild food restriction triggers relevant seasonal behavioral responses in advance of more lengthy physiological changes in an environment in which supplementary cues are salient. We compared the effects of FR treatment on Siberian hamster aggressive behavior, reproductive behavior, and fertility in either a long-day, summer-like photoperiod, in 
which modulation of food resources does not initiate seasonal changes, or an intermediate photoperiod, in which restricting food provides a signal that resource-limited conditions of winter are imminent. We hypothesized that hamsters in the ID-FR treatment would show modulation of behavior and reproduction. Our hypothesis was supported in our measurements of aggressive behavior, but not in reproductive behaviors or fertility. IDFR animals of both sexes displayed significantly increased aggression after just one week of treatments, but reproduction was unaffected throughout the study timeline.

Our finding that hamsters increase aggressive behavior in response to food availability as a supplementary cue provides important insight into the ultimate function of aggressive behavior in this and similar species. Most animals living in temperate climates display increased aggressive behavior/territoriality during the breeding season, coinciding with increased circulation of gonadal steroids (reviewed in: Monaghan \& Glickman 1992; Wingfield et al. 2006). It is relatively unusual to observe the opposite pattern, i.e., increased aggression during the non-breeding season, which exists in Siberian hamsters as well as other seasonally breeding species such as Syrian hamsters (Mesocricetus auratus), red squirrels (Tamiasciurus hudsonicus), and song sparrows (Melospiza melodia morphna) (Garrett \& Campbell 1980; Soma \& Wingfield 2001; Boonstra et al. 2008). One hypothesis for the adaptive function of this unusual behavioral pattern is that these animals become aggressive during the non-breeding season in order to defend limited food resources; this hypothesis is supported by our findings. In the present study, we provided FR hamsters with a modest $10 \%$ reduction in their normal ad libitum intake for a single week, and aggressive behavior increased only in combination with ID photoperiod treatment. This indicates that a signal of diminishing resources over 
a short time is sufficient to induce changes in behavior, but only in conditions that necessitate a specific seasonal response (i.e. intermediate photoperiod, and not long-day photoperiod). This provides an adaptive explanation for seasonal aggression by demonstrating that hamsters increase aggression in response to short-term restricted food availability, likely in order to defend limited food resources present during the short days of winter.

Because aggression during the non-breeding season is less common among animals, and occurs in opposition to seasonal patterns of gonadal steroid circulation, the endocrine mechanisms underlying this phenomenon are an area of active research. Notably, the adrenal androgen DHEA (a gonadal steroid precursor) has emerged as an important correlate of non-breeding season aggression in several species (Soma \& Wingfield 2001; Boonstra et al. 2008; Rendon et al. 2015a; Soma et al. 2015; Rendon \& Demas 2016). In Siberian hamsters specifically, our group has demonstrated a "seasonal shift" in endocrine mechanisms supporting aggression from gonadal steroids in the breeding season to the adrenal androgen DHEA in the non-breeding season (Rendon et al. 2015a). This shift allows for a decoupling of aggressive behavior from hormonal mechanisms underlying reproduction, which would be adaptive for species that must maintain aggression for other functions (i.e., defense of limited food resources) during times of reproductive quiescence.

In contrast to aggressive behavior, reproductive behavior was less malleable to treatment. ID-FR males have been shown to undergo gonadal regression after several weeks (Paul et al. 2009a; Paul et al. 2009b; Bailey et al. in review); our goal for this study was to assess potential changes in behavior that occur in advance of this lengthy 
physiological process, with the prediction that reproductive behavior would be impaired in ID-FR hamsters, particularly in females. Unexpectedly, we observed virtually no effects of treatment on female behavior and the opposite relationship in timing between behavior and physiology than we predicted in males. Male gonadal regression was already underway at the end of our 2-week behavioral observation period, but reproductive behaviors did not vary across treatments. By monitoring females for resulting pregnancies, we also determined that fertility was not affected by treatment, with pregnancy rate, littering rate, and litter characteristics all being equivalent across treatments.

The timing of changes in reproductive behavior and fertility during the response to short-day photoperiod has been examined in Syrian hamsters, a related species of seasonally breeding rodents (Beery et al. 2007). The study showed interesting individual variation in loss of reproductive behavior and fertility as well as sex differences: males took twice as long to reach a $50 \%$ siring rate as it took females to reach a $50 \%$ littering rate (Beery et al. 2007). Reproductive behavior also appeared decoupled from fertility, with females continuing to display receptivity even after loss of fertility and males continuing to mount for the entire 10-week duration (Beery et al. 2007). Our study addressed responses to a supplementary environmental cue (as opposed to photoperiod) in a different species, and our results support the findings of Beery et al. (2007). We predicted that behavior would exhibit more flexibility than physiology in response to seasonal cues, but reproductive behavior was well maintained in the face of inhibitory cues in both sexes, and ongoing physiological changes in males. It is presumably advantageous for males to preserve reproductive behaviors for longer, especially if 
matings with the declining proportion of fertile females can be attained over time. This is evidenced by male rodents continuing to exhibit reproductive behaviors even after castration (Meisel \& Sachs 1994), and is plausibly an adaptive strategy because of mechanisms such as the storage of viable sperm in the epididymis after the seasonal cessation of spermatogenesis (Jones 1999).

Although reproductive behavior and subsequent fertility were equivalent across treatments according to most of our measures, there were some notable exceptions. First, untreated male intruders were less likely to ejaculate when paired with an ID female. In addition to negatively impacting our sample (ID female groups ultimately consisted of 4 $(\mathrm{AL})$ and 2 (FR) females that were paired with an ejaculating male for pregnancy analysis), this observation has the potential to suggest that some mechanisms of nonvisible communication (Bradbury \& Vehrencamp 1998; Johnston 2003; Sales 2010) may differ according to photoperiod. Siberian hamsters communicate with olfactory cues through multiple secretions such as urine, feces, ventral glands, and sacculi glands (Ross 1998; Burger et al. 2001a; Burger et al. 2001b; Wynne-Edwards et al. 1992). Urinary (Rendon et al. 2016a) and ventral gland (Rendon et al. 2016b) compounds vary across photoperiod, but only in males. Siberian hamsters also produce ultrasonic vocalizations (USVs) that differ according to photoperiod and sex during aggressive contexts, and likely use them in other contexts as well, potentially including reproduction (Keesom et al. 2015; Rendon et al. 2015b). It is possible that these types of communication were employed during these reproductive interactions, and although ID females that were successfully inseminated during the encounter all produced litters, it may be meaningful that most receptive females in the ID photoperiod were not successfully inseminated. 
Differences in non-visual communication could also explain the effect we observed for FR males to spend more time investigating the ano-genital region of female intruders.

The second exception to our finding that reproduction seems to be robust to inhibitory supplementary cues is that, contrary to the male intruders, males in the ID-FR treatment were most likely to ejaculate during their encounter with a receptive female. This did not result in fertility consequences, as they were equally likely to have inseminated their female as males in other groups, but is interesting to note, especially considering that these males were already undergoing gonadal regression. This again reflects previous observations in Syrian hamsters, in which gonadally regressing males continued to mount throughout their exposure to short-day photoperiod (Beery et al. 2007). The ID-FR males being the most likely to ejaculate, and not merely less able to ejaculate than the other groups is reminiscent of the theorized terminal investment strategy observed in animals experiencing extreme stress or age, in which reproductive activity increases in response to situations that otherwise would call for conservation of energy in order to maximize lifetime fitness before death (Clutton-Brock 1984). It is possible that seasonally breeding males adopt this strategy on a seasonal timeframe when gonadal regression is underway, in order to attempt to reproduce before seasonal cessation of spermatogenesis. This idea has yet to be explored in detail, but there has been at least one suggestion that a type of seasonal terminal investment strategy takes place in male Siberian hamsters exposed to an immune challenge during gonadal regression (Weil et al. 2006). Weil et al. (2006) found that the process of gonadal regression was slowed in immune-challenged males, potentially to maximize reproductive activity in the face of uncertain survival to the next breeding season. 
Finally, investment in reproductive tissues differed across females, with ID females having smaller uterine horns compared to LD females. Although ID females performed competent reproductive behaviors, and all that were inseminated produced successful litters, the ID photoperiod treatment may have affected investment in reproductive tissues compared to females in the LD photoperiod. Because uterine horns were collected after the pregnancy period (which included increasing FR rations) from both pregnant and non-pregnant females, it is difficult to say whether food treatment would have affected female investment in reproductive tissues at another time point. However, this difference may be an important physiological underpinning of any possible communication by the ID females to their male intruders that resulted in a lower ejaculation rate.

\section{Conclusions}

Overall, we have demonstrated that food availability as a supplementary cue triggers seasonal aggressive, but not reproductive, responses in male and female Siberian hamsters over a short time period. These findings are consistent with the hypothesis that seasonal changes in aggression in this species are likely adaptive for defending limited food resources during specific times of the year (e.g., winter). The underlying mechanisms of seasonal aggression in this species, most importantly the ability of the adrenal androgen DHEA to drive seasonal changes in aggression, likely support this function by decoupling aggression from circulating gonadal steroids, which are reduced throughout the process of gonadal regression. In contrast, reproduction was robust to inhibitory environmental signals in the short term, potentially indicating a strategy for 
seasonally breeding animals to maximize reproduction prior to seasonal reproductive

quiescence. Collectively, our results provide important insight into how seasonally breeding animals coordinate important behavioral and physiological responses to relevant seasonal cues. Future study of animals that display seasonal changes in behavior and physiology should continue to investigate how endocrine mechanisms as well as nonvisual communication mechanisms between individuals support these changes according to the environment in order to maximize survival and reproductive success.

\section{Acknowledgments}

We thank Lizeth Achury, Andrea Amez, Jesus Bazan, Paola Guardado Flores, Emma St. John, and Ellen Weigel for assistance with laboratory procedures, Dr. Ned Place for generously providing a protocol for implantation site staining, and Dr. Vicky Meretsky for statistics consultation.

\section{Funding Sources}

This work was supported by the National Science Foundation [grant number IOS0543798 and a Graduate Research Fellowship to A.M.B and N.M.R], National Institutes of Health [training grant number T32HD049336] and Indiana University. These funding sources had no involvement in study design; in the collection, analysis and interpretation of data; in the writing of the report; or in the decision to submit this article for publication. 


\section{References}

Archer, J. (2006). Testosterone and human aggression: an evaluation of the challenge hypothesis. Neuroscience and Biobehavioral Reviews, 30(3), 319-345. doi:10.1016/j.neubiorev.2004.12.007

Baker, J. R. (1938). The evolution of breeding seasons. In: deBeer, G. R., ed. Essays on Aspects of Evolutionary Biology. Oxford, UK: Oxford University Press. 161-177 pp.

Bartness, T. J., Powers, J. B., Hastings, M. H., Bittman, E. L., \& Goldman, B. D. (1993). The timed infusion paradigm for melatonin delivery: What has it taught us about the melatonin signal, its reception, and the photoperiodic control of seasonal responses? Journal of Pineal Research, 15(4), 161-190.

Beery, A. K., Trumbull, J. J., Tsao, J. M., Costantini, R. M., \& Zucker, I. (2007). Sex differences in the onset of seasonal reproductive quiescence in hamsters. Proceedings of the Royal Society B-Biological Sciences, 274(1607), 281-286. doi:10.1098/rspb.2006.3726

Boonstra, R., Lane, J. E., Boutin, S., Bradley, A., Desantis, L., Newman, A. E. M., \& Soma, K. K. (2008). Plasma DHEA levels in wild, territorial red squirrels: Seasonal variation and effect of ACTH. General and Comparative Endocrinology, 158(1), 61-67. doi:10.1016/j.ygcen.2008.05.004

Bradbury, J. W. \& Vehrencamp, S. L. (1998). Principles of animal communication Sunderland, MA: Sinauer Associates, Inc.

Bronson, F. H. (1985). Mammalian reproduction: An ecological perspective. Biology of Reproduction, 32(1), 1-26. doi:10.1095/biolreprod32.1.1 
Bronson, F. H., \& Heideman, P. D. (1994). Seasonal regulation of reproduction in mammals. In: Knobil, E. and Neill, J. D., ed. The Physiology of Reproduction, Second Edition New York: Raven Press, Ltd. 541-583 pp.

Burger, B. V., Smit, D., Spies, H. S. C., Schmidt, C., Schmidt, U., Telitsina, A. Y., \& Grierson, G. R. (2001a). Mammalian exocrine secretions XV. Constituents of secretion of ventral gland of male dwarf hamster, Phodopus sungorus sungorus. Journal of Chemical Ecology, 27(6), 1259-1276. doi:10.1023/a:1010328331891

Burger, B. V., Smit, D., Spies, H. S. C., Schmidt, C., Schmidt, U., \& Telitsina, A. Y. (2001b). Mammalian exocrine secretions XVI. Constituents of secretion of supplementary sacculi of dwarf hamster, Phodopus sungorus sungorus. Journal of Chemical Ecology, 27(6), 1277-1288. doi:10.1023/a:1010380315961

Clutton-Brock, T. H. (1984). Reproductive effort and terminal investment in iteroparous animals. American Naturalist, 123(2), 212-229. doi:10.1086/284198

Desy, E. A., Batzli, G. O., \& Liu, J. (1990). Effects of food and predation on behavior of prairie voles: a field experiment. Oikos, 58(2), 159-168. doi:10.2307/3545423

Dodge, J. C., Kristal, M. B., \& Badura, L. L. (2002). Male-induced estrus synchronization in the female Siberian hamster (Phodopus sungorus sungorus). Physiology \& Behavior, 77(2-3), 227-231. doi:10.1016/s0031-9384(02)00851-x

Erb, G. E., Edwards, H. E., Jenkins, K. L., Mucklow, L. C., \& Wynne-Edwards, K. E. (1993). Induced components in the spontaneous ovulatory cycle of the Djungarian hamster (Phodopus campbelli). Physiology \& Behavior, 54(5), 955-959. doi:10.1016/0031-9384(93)90308-3 
Foltz, S. L., Ross, A. E., Laing, B. T., Rock, R. P., Battle, K. E., \& Moore, I. T. (2015). Get off my lawn: increased aggression in urban song sparrows is related to resource availability. Behavioral Ecology, 26(6), 1548-1557. doi:10.1093/beheco/arv111

French, S. S., Chester, E. M., \& Demas, G. E. (2016). Timing of maternal immunization affects immunological and behavioral outcomes of adult offspring in Siberian hamsters (Phodopus sungorus). Journal of Experimental Zoology Part aEcological Genetics and Physiology, 325(6), 377-389. doi:10.1002/jez.2023

Garrett, J. W., \& Campbell, C. S. (1980). Changes in social behavior of the male golden hamster accompanying photoperiodic changes in reproduction. Hormones and Behavior, 14(4), 303-318. doi:10.1016/0018-506x(80)90020-3

Goldman, B. D. (2001). Mammalian photoperiodic system: Formal properties and neuroendocrine mechanisms of photoperiodic time measurement. Journal of Biological Rhythms, 16(4), 283-301. doi:10.1177/074873001129001980

Hoffmann, K. (1982). The critical photoperiod in the Djungarian hamster Phodopus sungorus. In: Aschoff, J., Daan, S., \& Groos, G. A., ed. Vertebrate Circadian Systems: Structures and Physiology Berlin: Springer Verlag. 297-304 pp.

Jasnow, A. M., Huhman, K. L., Bartness, T. J., \& Demas, G. E. (2000). Short-day increases in aggression are inversely related to circulating testosterone concentrations in male Siberian hamsters (Phodopus sungorus). Hormones and Behavior, 38(2), 102-110. doi:10.1006/hbeh.2000.1604 
Johnston, R. E. (2003). Chemical communication in rodents: From pheromones to individual recognition. Journal of Mammalogy, 84(4), 1141-1162. doi:10.1644/BLe-010

Jones, R. C. (1999). To store or mature spermatozoa? The primary role of the epididymis. International Journal of Andrology, 22(2), 57-67.

Keesom, S. M., Rendon, N. M., Demas, G. E., \& Hurley, L. M. (2015). Vocal behaviour during aggressive encounters between Siberian hamsters, Phodopus sungorus. Animal Behaviour, 102, 85-93. doi:10.1016/j.anbehav.2015.01.014

Kopf, R., Lorenz, D., \& Salewski, E. (1964). The influence of thalidomide on the fertility of rats in the reproduction experiment over two generations. [Der Einfluss von Thalidomid aur die Fertilitat von Ratten im Generation-sversuch fiber zwei Generationen]. Arch Exp Pathol Pharmakol [Naunyn Schmiede Bergs], 247(2), $121-135$.

Lincoln, G. A., Andersson, H., \& Loudon, A. (2003). Clock genes in calendar cells as the basis of annual timekeeping in mammals - a unifying hypothesis. Journal of Endocrinology, 179(1), 1-13. doi:10.1677/joe.0.1790001

Lore, R., Gottdiener, C., \& Delahunty, M. J. (1986). Lean and mean rats: some effects of acute changes in the food supply upon territorial aggression. Aggressive Behavior, 12(6), 409-415.

doi:10.1002/1098-2337(1986)12:6<409::aid-ab2480120603>3.0.co;2-6

Maruyama, A., Rusuwa, B., \& Yuma, M. (2010). Asymmetric interspecific territorial competition over food resources amongst Lake Malawi cichlid fishes. African Zoology, 45(1), 24-31. doi:10.3377/004.045.0116 
McMillan, H. J., \& Wynne-Edwards, K. E. (1999). Divergent reproductive endocrinology of the estrous cycle and pregnancy in dwarf hamsters (Phodopus). Comparative Biochemistry and Physiology A-Molecular and Integrative Physiology, 124(1), 53-67. doi:10.1016/s1095-6433(99)00090-2

Meisel, R. L., \& Sachs, B. D. (1994). The physiology of male sexual behavior. In: Knobil, E. \& Neill, J. D., ed. The Physiology of Reproduction, Second Edition New York: Raven Press, Ltd. 3-105 pp.

Monaghan, E. P., \& Glickman, S. E. (1992). Hormones and aggressive behavior. In: Becker, J. B., Breedlove, S. M., \& Crews, D., ed. Behavioral Endocrinology Cambridge, MA: MIT Press. 261-285 pp.

Paul, M. J., Galang, J., Schwartz, W. J., \& Prendergast, B. J. (2009a). Intermediateduration day lengths unmask reproductive responses to nonphotic environmental cues. American Journal of Physiology-Regulatory Integrative and Comparative Physiology, 296(5), R1613-R1619. doi:10.1152/ajpregu.91047.2008

Paul, M. J., Pyter, L. M., Freeman, D. A., Galang, J., \& Prendergast, B. J. (2009b). Photic and nonphotic seasonal cues differentially engage hypothalamic kisspeptin and RFamide-related peptide mRNA expression in Siberian hamsters. Journal of Neuroendocrinology, 21(12), 1007-1014. doi:10.1111/j.1365-2826.2009.01924.x

Rendon, N. M., \& Demas, G. E. (2016). Bi-directional actions of dehydroepiandrosterone and aggression in female Siberian hamsters. Journal of Experimental Zoology Part a-Ecological Genetics and Physiology, 325(2), 116-121. doi:10.1002/jez.2001 
Rendon, N. M., Rudolph, L. M., Sengelaub, D. R., \& Demas, G. E. (2015a). The agonistic adrenal: melatonin elicits female aggression via regulation of adrenal androgens. Proceedings of the Royal Society B-Biological Sciences, 282(1819). doi:10.1098/rspb.2015.2080

Rendon, N. M., Keesom, S. M., Amadi, C., Hurley, L. M., \& Demas, G. E. (2015b). Vocalizations convey sex, seasonal phenotype, and aggression in a seasonal mammal. Physiology \& Behavior, 152, 143-150. doi:10.1016/j.physbeh.2015.09.014

Rendon, N. M., Soini, H. A., Scotti, M.-A. L., Weigel, E. R., Novotny, M. V., \& Demas, G. E. (2016b). Photoperiod and aggression induce changes in ventral gland compounds exclusively in male Siberian hamsters. Hormones and Behavior, 81, 1-11. doi:http://dx.doi.org/10.1016/j.yhbeh.2016.02.005

Rendon, N. M., Soini, H.A., Scotti, M-A.L., Novotny, M.V., Demas, G.E. . (2016a). Urinary volatile compounds differ across reproductive phenotypes and following aggression in male Siberian hamsters. Physiology \& Behavior, 164, 58-67.

Roosa, K. A., Zysling, D. A., \& Place, N. J. (2015). An assessment of anti-Mullerian hormone in predicting mating outcomes in female hamsters that have undergone natural and chemically-accelerated reproductive aging. General and Comparative Endocrinology, 214, 56-61. doi:10.1016/j.ygcen.2015.03.007

Ross, P. D. (1998). Phodopus sungorus. Mammalian Species, 595, 1-9.

Sales, G. D. (2010). Ultrasonic calls of wild and wild-type rodents. In Brudzynski, S. M., ed. Handbook of Mammalian Vocalization: An Integrative Neuroscience Approach Vol. 19 Amsterdam, Netherlands: Elsevier Science BV. 77-88 pp. 
Scotti, M.-A. L., Place, N. J., \& Demas, G. E. (2007). Short-day increases in aggression are independent of circulating gonadal steroids in female Siberian hamsters (Phodopus sungorus). Hormones and Behavior, 52(2), 183-190. doi:10.1016/j.yhbeh.2007.03.029

Scribner, S. J., \& Wynne-Edwards, K. E. (1994). Disruption of body temperature and behavior rhythms during reproduction in dwarf hamster (Phodopus). Physiology \& Behavior, 55(2), 361-369. doi:10.1016/0031-9384(94)90147-3

Soma, K. K., Rendon, N. M., Boonstra, R., Albers, H. E., \& Demas, G. E. (2015). DHEA effects on brain and behavior: Insights from comparative studies of aggression. Journal of Steroid Biochemistry and Molecular Biology, 145, 261-272. doi:10.1016/j.jsbmb.2014.05.011

Soma, K. K., \& Wingfield, J. C. (2001). Dehydroepiandrosterone in songbird plasma: Seasonal regulation and relationship to territorial aggression. General and Comparative Endocrinology, 123(2), 144-155. doi:10.1006/gcen.2001.7657

Weil, Z. M., Martin, L. B., Workman, J. L., \& Nelson, R. J. (2006). Immune challenge retards seasonal reproductive regression in rodents: evidence for terminal investment. Biology Letters, 2(3), 393-396. doi:10.1098/rsbl.2006.0475

Wingfield, J. C., \& Farner, D. S. (1980). Control of seasonal reproduction in temperate zone birds. In: Reiter, R. J. \& Follett, B. K., ed. Progress in Reproductive Biology: Seasonal Reproduction in Higher Vertebrates New York: S. Karger. 62$101 \mathrm{pp}$.

Wingfield, J. C., Ball, G. F., Dufty, A. M., Hegner, R. E., \& Ramenofsky, M. (1987). Testosterone and aggression in birds. American Scientist, 75(6), 602-608. 
Wingfield, J. C., Moore, I. T., Goymann, W., Wacker, D. W., \& Sperry, T. (2006). Contexts and ethology of vertebrate aggression: implications for the evolution of hormone-behavior interactions. In: Nelson, R. J., ed. The Biology of Aggression New York: Oxford University Press. 179-210 pp.

Wynne-Edwards, K. E., Surov, A. V., \& Telitzina, A. Y. (1992). Field studies of chemical signaling: direct observations of dwarf hamsters (Phodopus) in soviet Asia. In: Doty, R. L. \& Müller-Schwarze, D., ed. Chemical Signals in Vertebrates VI New York: Plenum Press. 485-491 pp. 